International Journal of Pure and Applied Mathematics

Volume 105 No. 4 2015, 763-773

ISSN: 1311-8080 (printed version); ISSN: 1314-3395 (on-line version)

url: http://www.ijpam.eu

doi: http://dx.doi.org/10.12732/ijpam.v105i4.16

ijpam.eu

\title{
ON THE SMOOTNESS OF FUNCTION OF REACHABILITY FOR AUTONOMOUS DIFFERENTIAL EQUATIONS
}

\author{
K.G. Dishlieva \\ Department of Differential Equations \\ Faculty of Applied Mathematics and Informatics \\ Technical University of Sofia \\ 1756 Sofia, 8 Kl. Ohridski Blvd, BULGARIA
}

\begin{abstract}
A function of reachability $\Theta^{+}$is investigated for a wide class of autonomous systems of differential equations and relatively wide class reachable sets. The sufficient conditions for differentiability of the function $\Theta^{+}$are found.
\end{abstract}

AMS Subject Classification: $34 \mathrm{~A} 34$

Key Words: autonomous differential equations, function of reachability, differentiability

\section{Introduction}

Assume that:

- The domain $G \subset R^{n}$ is a phase space of autonomous system of differential equations;

- The initial point $x_{0} \in G$;

- The set $\Phi \neq \emptyset$;

- The curve $\gamma, \gamma \subset G$, is a trajectory of the autonomous system which:

- at the moment $t_{0}=0$, passes through the initial point $x_{0}$;

- crosses the set $\Phi$, i.e. $\gamma \cap \Phi \neq \emptyset$.

Received: September 28, 2015

(c) 2015 Academic Publications, Ltd. url: www.acadpubl.eu 
We say that $\Theta^{+}$is a function of reachability if this function assigning to the initial point $x_{0}$ the moment $\theta$ at which the trajectory $\gamma$ crosses $\Phi$ for the first time. The articles [7], [8] and [14] are devoted on the qualities of function of reachability. The sufficient conditions under which the function is continuous and bounded are found there. In this paper, the studies of function qualities are continued. The sufficient conditions for differentiability of $\Theta^{+}$in respect to the initial point $x_{0}$ are given.

The function of reachability has a fundamental role in the studies on the existence of periodic solutions of the systems of differential equations with variable moments of the impulsive effects. (see [6] and [13]). In the definitions of impulsive equations, the set of reachability is a set of impulses too. The impulsive effects take place when the trajectory of impulsive system reaches the set $\Phi$. The trajectory instantaneously "bounces" at the moment of these impacts. Thus, the study of function of reachability is equivalent to the investigation of impulsive moments of the systems of differential equations with impulsive effects.

The dynamic processes that have interrupted nature can be modeled by the impulsive systems of differential equations (see [1]-[5] and [10]-[11]). The processes in which the impulsive effects are not predefined, and they are performed when the trajectory reaches the above-mentioned impulsive sets are particularly important. In these cases, the function of reachability is an important feature of such processes and their corresponding models.

The considerations above justify the research presented in this paper.

\section{Preliminary Remarks}

The scalar product and Euclidean norm in $R^{n}$ are denoted by $\langle x, y\rangle$ and $\|x\|$, respectively. For the points $x=\left(x_{1}, x_{2}, \ldots, x_{n}\right)$ and $y=\left(y_{1}, y_{2}, \ldots, y_{n}\right)$ in $R^{n}$, we have

$$
\begin{aligned}
\langle x, y\rangle & =x_{1} y_{1}+x_{2} y_{2}+\cdots+x_{n} y_{n}, \\
\|x\| & =(\langle x, x\rangle)^{1 / 2}=\left(\left(x_{1}\right)^{2}+\left(x_{2}\right)^{2}+\cdots+\left(x_{n}\right)^{2}\right)^{\frac{1}{2}} .
\end{aligned}
$$

We will use the unit vectors:

$$
e_{1}=(1,0, \ldots, 0), e_{2}=(0,1,0, \ldots, 0), \ldots, e_{n}(0,0, \ldots, 1) \in R^{n} .
$$

The closure and contour of the set $X$ are denoted by $\bar{X}$ and $\partial X$, correspondingly. Euclidean distance between the sets $X$ and $Y$ will denote by $\rho(X, Y)$. 
The main object of investigation is the following initial value problem

$$
\frac{d x}{d t}=f(x), \quad x(0)=x_{0},
$$

where: the function $f: G \rightarrow R^{n}$; set $G \subset R^{n}, G \neq \emptyset$ and $G$ is a domain (an open and connected set); the initial point $x_{0} \in G$. The solution of problem (1) will be denoted by $x\left(t ; x_{0}\right)$. The trajectory of system (1), closed between the points $x\left(0 ; x_{0}\right)=x_{0}$ and $x\left(\theta ; x_{0}\right)$, where $\theta \in R$ will be denoted by $\gamma\left(\theta, x_{0}\right)$. We have

$$
\gamma\left(\theta, x_{0}\right)= \begin{cases}\left\{x=x\left(t ; x_{0}\right) ; 0 \leq t<\theta\right\}, & 0<\theta ; \\ \left\{x=x\left(t ; x_{0}\right) ; \theta<t \leq 0\right\}, & \theta<0 .\end{cases}
$$

In particular:

$$
\gamma\left(\infty, x_{0}\right)=\left\{x=x\left(t ; x_{0}\right) ; 0 \leq t<\infty\right\}
$$

and

$$
\gamma\left(-\infty, x_{0}\right)=\left\{x=x\left(t ; x_{0}\right) ;-\infty<t \leq 0\right\} .
$$

Definition 1. [8] Assume that:

1. The sets $X_{0}^{+} \subset G, \Phi \subset G, X_{0}^{+} \neq \emptyset$ and $\Phi \neq \emptyset$;

2. For any initial point $x_{0} \in X_{0}^{+}$, the solution of problem (1) is defined and unique in the infinite interval $[0, \infty)$;

3. There exists a function $\Theta^{+}: X_{0}^{+} \rightarrow R^{+}$, which assigns to any point $x_{0} \in X_{0}^{+}$a positive constant $\theta=\Theta^{+}\left(x_{0}\right)$ for which it is satisfied:

- $x\left(\theta ; x_{0}\right) \in \Phi$

- $x\left(t ; x_{0}\right) \notin \Phi$ for $0 \leq t<\theta$.

Then, we say that:

1. $\Phi$ is a positive reachable set from the set $X_{0}^{+}$through the system (1);

2. $X_{0}^{+}$is a positive initial set for $\Phi$ through the system (1);

3. Any initial point $x_{0} \in X_{0}^{+}$is named positive initial point for $\Phi$ through the system (1);

4. If $X_{0}^{+}=G$, then $\Phi$ is a totally positive reachable set through the system (1); 
5. The function $\Theta^{+}: X_{0}^{+} \rightarrow R^{+}$is named a positive function of reachability.

The following concepts are defined in a similar manner:

1. Negative reachable set;

2. Negative initial set $X_{0}^{-}$;

3. Negative initial point;

4. Totally negative reachable set;

5. Negative function of reachability $\Theta^{-}: X_{0}^{-} \rightarrow R^{-}$.

Let the reachable set has the form

$$
\Phi=\{x \in G ; \varphi(x)=0\}
$$

where the function $\varphi: G \rightarrow R$.

We introduce the following conditions:

H1. There exists a constant $C_{f}>0$ such that

$$
(\forall x \in G) \Rightarrow\|f(x)\| \leq C_{f}
$$

H2. There exists a constant $C_{\text {Lip }}>0$ such that

$$
\left(\forall x^{\prime}, x^{\prime \prime} \in G\right) \Rightarrow\left\|f\left(x^{\prime}\right)-f\left(x^{\prime \prime}\right)\right\| \leq C_{L i p}\left\|x^{\prime}-x^{\prime \prime}\right\|
$$

H3. For any point $x_{0} \in G$, the solution of problem (1) exists and is unique in $R$.

H4. The function $\varphi \in C^{1}[G, R]$. There exists a constant $C_{\langle\operatorname{grad} \varphi, f\rangle}>0$ such that

$$
(\forall x \in \Phi) \Rightarrow\langle\operatorname{grad} \varphi(x), f(x)\rangle \geq C_{\langle\operatorname{grad} \varphi, f\rangle} .
$$

H5. The set $\Phi$ is connected.

H6. It is fulfilled $\bar{\Phi} \backslash \Phi \subset \partial G$.

H7. There exists a constant $C_{\varphi}>0$ such that

$$
(\forall x \in G) \Rightarrow|\varphi(x)| \leq C_{\varphi} \rho(x, \Phi) .
$$

Theorem 1. [7], [8]. Let the conditions $H 1-H 7$ be valid.

Then:

1. If the point $x_{0} \in X_{0}^{-}$, then the trajectory $\gamma\left(\Theta^{-}\left(x_{0}\right) ; x_{0}\right) \subset X_{0}^{-}$; 
2. If the point $x_{0} \in X_{0}^{+}$, then the trajectory $\gamma\left(\Theta^{+}\left(x_{0}\right) ; x_{0}\right) \subset X_{0}^{+}$;

3. If the point $x_{0} \in X_{0}^{-}$, then the trajectory $\gamma\left(\infty ; x_{0}\right) \subset X_{0}^{-}$;

4. If the point $x_{0} \in X_{0}^{+}$, then the trajectory $\gamma\left(-\infty ; x_{0}\right) \subset X_{0}^{+}$;

5. The sets $X_{0}^{-} \neq \emptyset$ and $X_{0}^{+} \neq \emptyset$;

6. The set $\Phi \subset \overline{X_{0}^{-}}$and $\Phi \subset \overline{X_{0}^{+}}$;

7. The sets $X_{0}^{-}$and $X_{0}^{+}$are open;

8. The set $X_{0}=X_{0}^{-} \cup X_{0}^{+} \cup \Phi$ is open;

9. The set $X_{0}$ is connected;

10. The functions $\Theta^{-} \in C\left[X_{0}^{-}, R^{-}\right]$and $\Theta^{+} \in C\left[X_{0}^{+}, R^{+}\right]$.

Let us consider the particular case when the reachable set $\Phi$ is a part of one hyperplane, i.e.

$$
\left(\exists a=\left(a_{1}, a_{2}, \ldots, a_{n}\right) \in R^{n}, a \neq 0\right)\left(\exists a_{0} \in R\right): \Phi=\left\{x \in G,\langle a, x\rangle+a_{0}=0\right\} .
$$

In other words, the function $\varphi(x)=\langle a, x\rangle+a_{0}, x \in G$.

In connection with this particular case, we introduce the following condition. H8. There exists a constant $C_{\langle a, f\rangle}>0$ such that

$$
\left(\forall x \in \Phi \Leftrightarrow\langle a, x\rangle+a_{0}=0, x \in G\right) \Rightarrow\langle a, f(x)\rangle \geq C_{\langle a, f\rangle} .
$$

As a consequence of the previous theorem (in the case where the reachable set is a part of the hyperplane), we formulate the following statement.

Corollary 1. Let:

1. The function $\varphi(x)=\langle a, x\rangle+a_{0}, x \in G$;

2. The conditions H1-H3 and H8 hold.

Then the assertions of Theorem 1 are satisfied.

Proof. We will check whether the conditions of Theorem 1 are valid, which is the evidence of this corollary. It is clear that the function $\left(\varphi(x)=\langle a, x\rangle+a_{0}\right) \in$ $C^{1}[G, R]$. Furthermore, by using the condition $\mathrm{H} 8$, we find

$$
(\forall x \in \Phi) \Rightarrow\langle\operatorname{grad} \varphi(x), f(x)\rangle=\langle a, f(x)\rangle \geq C_{\langle a, f\rangle}=C_{\langle\operatorname{grad} \varphi, f\rangle},
$$

i.e. the condition $\mathrm{H} 4$ is fulfilled. The conditions $\mathrm{H} 5$ and $\mathrm{H} 6$ are checked trivial. In this case, we have

$$
\begin{aligned}
(\forall x \in G) \Rightarrow|\varphi(x)| & =\left|\langle a, x\rangle+a_{0}\right|=\frac{\left|\langle a, x\rangle+a_{0}\right|}{\|a\|} \cdot\|a\| \\
& =\|a\| . \rho(x, \Phi)=C_{\varphi} \cdot \rho(x \cdot \Phi),
\end{aligned}
$$


by which the condition $\mathrm{H} 7\left(C_{\varphi}=\|a\|\right)$ is established.

Remark 1. Let the initial point of problem (1) be fixed and $x_{0}=\left(x_{01}, x_{02}\right.$, $\left.\ldots, x_{0 n}\right) \in X_{0}^{+}$. If we assume that the private derivatives

$$
\frac{\partial}{\partial x_{0 i}} \Theta^{+}\left(x_{0}\right), i=1,2, \ldots, n,
$$

exist, then we will show the equations that they satisfy.

Firstly, we will examine the general case where the function $\varphi \in C^{1}[G, R]$. For any point $x_{0}=\left(x_{01}, x_{02}, \ldots, x_{0 n}\right) \in X_{0}^{+}$, we have

$$
\begin{aligned}
\varphi\left(x\left(\Theta^{+}\left(x_{0}\right) ; x_{0}\right)\right)=0 \Leftrightarrow \quad & \varphi\left(x_{1}\left(\Theta^{+}\left(x_{01}, x_{02}, \ldots, x_{0 n}\right) ;\left(x_{01}, x_{02}, \ldots, x_{0 n}\right)\right),\right. \\
& x_{2}\left(\Theta^{+}\left(x_{01}, x_{02}, \ldots, x_{0 n}\right) ;\left(x_{01}, x_{02}, \ldots, x_{0 n}\right)\right), \ldots, \\
& \left.x_{n}\left(\Theta^{+}\left(x_{01}, x_{02}, \ldots, x_{0 n}\right) ;\left(x_{01}, x_{02}, \ldots, x_{0 n}\right)\right)\right) .
\end{aligned}
$$

Let us formally differentiate the equation above in respect to $x_{0 i}$, where $i \in$ $\{1,2, \ldots, n\}$. Consistently, we get:

$$
\begin{aligned}
& \frac{\partial}{\partial x_{1}} \varphi\left(x\left(\Theta^{+}\left(x_{0}\right) ; x_{0}\right)\right) \cdot \frac{\partial}{\partial x_{0 i}} x_{1}\left(\Theta^{+}\left(x_{0}\right) ; x_{0}\right) \\
& +\frac{\partial}{\partial x_{2}} \varphi\left(x\left(\Theta^{+}\left(x_{0}\right) ; x_{0}\right)\right) \cdot \frac{\partial}{\partial x_{0 i}} x_{2}\left(\Theta^{+}\left(x_{0}\right) ; x_{0}\right) \\
& +\ldots+ \\
& +\frac{\partial}{\partial x_{n}} \varphi\left(x\left(\Theta^{+}\left(x_{0}\right) ; x_{0}\right)\right) \cdot \frac{\partial}{\partial x_{0 i}} x_{n}\left(\Theta^{+}\left(x_{0}\right) ; x_{0}\right) \\
& \Leftrightarrow\left\langle\operatorname{grad} \varphi\left(x\left(\Theta^{+}\left(x_{0}\right) ; x_{0}\right)\right), \frac{\partial}{\partial x_{0 i}} x\left(\Theta^{+}\left(x_{0}\right) ; x_{0}\right)\right\rangle=0 \\
& \Leftrightarrow\left\langle\operatorname{grad} \varphi\left(x\left(\Theta^{+}\left(x_{0}\right) ; x_{0}\right)\right), \frac{\partial}{\partial x_{0 i}}\left(x_{0}+\int_{0}^{\Theta^{+}\left(x_{0}\right)} f\left(x\left(\tau ; x_{0}\right)\right) d \tau\right)\right\rangle=0 \\
& \Leftrightarrow\left\langle\operatorname{grad} \varphi\left(x\left(\Theta^{+}\left(x_{0}\right) ; x_{0}\right)\right),\right. \\
& \left.\left(e_{i}+\int_{0}^{\Theta^{+}\left(x_{0}\right)} \frac{\partial}{\partial x_{0 i}} f\left(x\left(\tau ; x_{0}\right)\right) d \tau+\frac{\partial}{\partial x_{0 i}} \Theta^{+}\left(x_{0}\right) \cdot f\left(x\left(\Theta^{+}\left(x_{0}\right) ; x_{0}\right)\right)\right)\right\rangle=0 \\
& \Leftrightarrow\left\langle\operatorname{grad} \varphi\left(x\left(\Theta^{+}\left(x_{0}\right) ; x_{0}\right)\right), \frac{\partial}{\partial x_{0 i}} \Theta^{+}\left(x_{0}\right) \cdot f\left(x\left(\Theta^{+}\left(x_{0}\right) ; x_{0}\right)\right)\right\rangle \\
& =-\left\langle\operatorname{grad} \varphi\left(x\left(\Theta^{+}\left(x_{0}\right) ; x_{0}\right)\right), e_{i}\right\rangle
\end{aligned}
$$




$$
-\left\langle\operatorname{grad} \varphi\left(x\left(\Theta^{+}\left(x_{0}\right) ; x_{0}\right)\right), \int_{0}^{\Theta^{+}\left(x_{0}\right)} \frac{\partial}{\partial x_{0 i}} f\left(x\left(\tau ; x_{0}\right)\right) d \tau\right\rangle,
$$

where $e_{1}=(1,0, \ldots, 0), e_{2}=(0,1,0, \ldots, 0), \ldots, e_{n}=(0,0, \ldots, 1)$. From the equation above, after trivial transformations, we obtain

$$
\begin{aligned}
& \frac{\partial}{\partial x_{0 i}} \Theta^{+}\left(x_{0}\right) \\
= & -\frac{\frac{\partial}{\partial x_{i}} \varphi\left(x\left(\Theta^{+}\left(x_{0}\right) ; x_{0}\right)\right)}{\left\langle\operatorname{grad} \varphi\left(x\left(\Theta^{+}\left(x_{0}\right) ; x_{0}\right)\right), f\left(x\left(\Theta^{+}\left(x_{0}\right) ; x_{0}\right)\right)\right\rangle} \\
& -\frac{\left\langle\operatorname{grad} \varphi\left(x\left(\Theta^{+}\left(x_{0}\right) ; x_{0}\right)\right), \int_{0}^{\Theta^{+}\left(x_{0}\right)} \frac{\partial}{\partial x} f\left(x\left(\tau ; x_{0}\right)\right) \cdot \frac{\partial}{\partial x_{0 i}} x\left(\tau ; x_{0}\right) d \tau\right\rangle}{\left\langle\operatorname{grad} \varphi\left(x\left(\Theta^{+}\left(x_{0}\right) ; x_{0}\right)\right), f\left(x\left(\Theta^{+}\left(x_{0}\right) ; x_{0}\right)\right)\right\rangle} .
\end{aligned}
$$

In the particular case, when the reachable set is a part of hyperplane, we find that

$$
\frac{\partial}{\partial x_{0 i}} \Theta^{+}\left(x_{0}\right)=-\frac{\left\langle a, e_{i}\right\rangle+\left\langle a, \int_{0}^{\Theta^{+}\left(x_{0}\right)} \frac{\partial}{\partial x} f\left(x\left(\tau ; x_{0}\right)\right) \cdot \frac{\partial}{\partial x_{0 i}} x\left(\tau ; x_{0}\right) d \tau\right\rangle}{\left\langle a, f\left(x\left(\Theta^{+}\left(x_{0}\right) ; x_{0}\right)\right)\right\rangle}
$$

The above formal equality allows us to formulate the main results in the present study.

\section{Main Results}

Theorem 2. Let:

1. The function $\varphi(x)=\langle a, x\rangle+a_{0}, x \in G$.

2. The conditions H1-H3 and H8 hold.

Then the function $\Theta^{+}: X_{0}^{+} \rightarrow R^{+}$is differentiable in respect to $x_{0}=$ $\left(x_{01}, x_{02}, \ldots, x_{0 n}\right) \in X_{0}^{+}$and the private derivatives

$$
\frac{\partial}{\partial x_{0 i}} \Theta^{+}\left(x_{0}\right), \quad i=1,2, \ldots, n,
$$

satisfy the corresponding equations (2).

Proof. Let $i \in\{1,2, \ldots, n\}$, i.e. $i$ is a fixed number. Since the positive initial set $X_{0}^{+} \neq \emptyset$, then there exists at least one initial point $x_{0}=\left(x_{01}, x_{02}, \ldots, x_{0 n}\right) \in$ 
$X_{0}^{+}$. As $X_{0}^{+}$is an open set, then

$$
\begin{gathered}
\left(\exists \Delta_{i}=\text { const }>0\right):\left(\forall \Delta x_{i} \in R, 0 \leq\left|\Delta x_{i}\right|<\Delta_{i}\right) \\
\Rightarrow x_{0}^{*}=\left(x_{01}, \ldots, x_{0 i-1}, x_{0 i}+\Delta x_{i}, x_{0 i+1}, \ldots, x_{0 n}\right) \in X_{0}^{+} .
\end{gathered}
$$

According to the definition, the next inequalities are fulfilled:

$$
\begin{aligned}
& \varphi\left(x\left(\Theta^{+}\left(x_{0}^{*}\right) ; x_{0}^{*}\right)\right)=\left\langle a, x\left(\Theta^{+}\left(x_{0}^{*}\right) ; x_{0}^{*}\right)\right\rangle+a_{0}=0 ; \\
& \varphi\left(x\left(\Theta^{+}\left(x_{0}\right) ; x_{0}\right)\right)=\left\langle a, x\left(\Theta^{+}\left(x_{0}\right) ; x_{0}\right)\right\rangle+a_{0}=0,
\end{aligned}
$$

from where, for any $\Delta x_{i} \in R, 0<\left|\Delta x_{i}\right|<\Delta_{i}$, we obtain

$$
\begin{aligned}
& \frac{1}{\Delta x_{i}}\left(\left\langle a, x\left(\Theta^{+}\left(x_{0}^{*}\right) ; x_{0}^{*}\right)\right\rangle+a_{0}-\left\langle a, x\left(\Theta^{+}\left(x_{0}\right) ; x_{0}\right)\right\rangle-a_{0}\right)=0 \\
\Leftrightarrow & \left\langle a, \frac{1}{\Delta x_{i}}\left(x\left(\Theta^{+}\left(x_{0}^{*}\right) ; x_{0}^{*}\right)-x\left(\Theta^{+}\left(x_{0}\right) ; x_{0}\right)\right)\right\rangle=0 \\
\Leftrightarrow & \left\langle a, \frac{1}{\Delta x_{i}}\left(x_{0}^{*}+\int_{0}^{\Theta^{+}\left(x_{0}^{*}\right)} f\left(x\left(\tau ; x_{0}^{*}\right)\right) d \tau-x_{0}-\int_{0}^{\Theta^{+}\left(x_{0}\right)} f\left(x\left(\tau ; x_{0}\right)\right) d \tau\right)\right\rangle=0 \\
\Leftrightarrow & \left\langle a, e_{i}\right\rangle+\left\langle a, \frac{1}{\Delta x_{i}}\left(\int_{0}^{\Theta^{+}\left(x_{0}^{*}\right)} f\left(x\left(\tau ; x_{0}^{*}\right)\right) d \tau-\int_{0}^{\Theta^{+}\left(x_{0}\right)} f\left(x\left(\tau ; x_{0}\right)\right) d \tau\right)\right\rangle=0 .
\end{aligned}
$$

Further, for convenience, we will assume that $\Theta^{+}\left(x_{0}\right) \leq \Theta^{+}\left(x_{0}^{*}\right)$. Then, from the last equality it follows:

$$
\begin{gathered}
\left\langle a, e_{i}\right\rangle+\left\langle a, \frac{1}{\Delta x_{i}}\left(\int_{0}^{\Theta^{+}\left(x_{0}\right)}\left(f\left(x\left(\tau ; x_{0}^{*}\right)\right)-f\left(x\left(\tau ; x_{0}\right)\right)\right) d \tau\right.\right. \\
\left.\left.+\int_{\Theta^{+}\left(x_{0}\right)}^{\Theta^{+}\left(x_{0}^{*}\right)} f\left(x\left(\tau ; x_{0}^{*}\right)\right) d \tau\right)\right\rangle=0 \\
\Leftrightarrow\left\langle a, e_{i}\right\rangle+\left\langle a, \int_{0}^{\Theta^{+}\left(x_{0}\right)} \frac{\partial}{\partial x} f\left(x\left(\tau ; x_{0}+\mu \cdot \Delta_{i} \cdot e_{i}\right)\right) \cdot \frac{1}{\Delta x_{i}}\left(x\left(\tau ; x_{0}^{*}\right)-x\left(\tau ; x_{0}\right)\right) d \tau\right\rangle \\
+\left\langle a, \frac{1}{\Delta x_{i}}\left(\Theta^{+}\left(x_{0}^{*}\right)-\Theta^{+}\left(x_{0}\right)\right) \cdot f\left(x\left(\theta ; x_{0}^{*}\right)\right)\right\rangle=0,
\end{gathered}
$$

where $0 \leq \mu \leq 1$ and $\Theta^{+}\left(x_{0}\right) \leq \theta \leq \Theta^{+}\left(x_{0}^{*}\right)$. Finally, we derive

$$
\frac{1}{\Delta x_{i}}\left(\Theta^{+}\left(x_{0}^{*}\right)-\Theta^{+}\left(x_{0}\right)\right)
$$




$$
\begin{aligned}
= & -\frac{\left\langle a, e_{i}\right\rangle}{\left\langle a, f\left(x\left(\theta ; x_{0}^{*}\right)\right)\right\rangle} \\
& -\frac{\left\langle a, \int_{0}^{\Theta^{+}\left(x_{0}\right)} \frac{\partial}{\partial x} f\left(x\left(\tau ; x_{0}+\mu . \Delta_{i} \cdot e_{i}\right)\right) \cdot \frac{1}{\Delta x_{i}}\left(x\left(\tau ; x_{0}^{*}\right)-x\left(\tau ; x_{0}\right)\right) d \tau\right\rangle}{\left\langle a, f\left(x\left(\theta ; x_{0}^{*}\right)\right)\right\rangle} .
\end{aligned}
$$

Bearing in mind the way in which the constants $\theta$ and $\mu$ are obtained, we deduce that if

$$
\Delta x_{i} \rightarrow 0 \Rightarrow \theta \rightarrow \Theta^{+}\left(x_{0}\right), \mu . \Delta x_{i} \rightarrow 0
$$

Then:

$$
\begin{aligned}
& \lim _{\Delta x_{i} \rightarrow 0} f\left(x\left(\theta ; x_{0}^{*}\right)\right)=\lim _{\theta \rightarrow \Theta^{+}\left(x_{0}\right)} \lim _{\Delta x_{i} \rightarrow 0} f\left(x\left(\theta ; x_{0}+\Delta x_{i} \cdot e_{i}\right)\right) \\
& =f\left(x\left(\Theta^{+}\left(x_{0}\right) ; x_{0}\right)\right) ; \\
& \lim _{\Delta x_{i} \rightarrow 0} \frac{\partial}{\partial x} f\left(x\left(\tau ; x_{0}+\mu \cdot \Delta_{i} . e_{i}\right)\right)=\lim _{\mu \cdot \Delta x_{i} \rightarrow 0} \frac{\partial}{\partial x} f\left(x\left(\tau ; x_{0}+\mu \cdot \Delta_{i} \cdot e_{i}\right)\right) \\
& =\frac{\partial}{\partial x} f\left(x\left(\tau ; x_{0}\right)\right) ; \\
& \lim _{\Delta x_{i} \rightarrow 0} \frac{1}{\Delta x_{i}}\left(x\left(\tau ; x_{0}^{*}\right)-x\left(\tau ; x_{0}\right)\right)=\lim _{\Delta x_{i} \rightarrow 0} \frac{1}{\Delta x_{i}}\left(x\left(\tau ; x_{0}+\Delta x_{i} \cdot e_{i}\right)-x\left(\tau ; x_{0}\right)\right) \\
& =\frac{\partial}{\partial x_{0 i}} x\left(\tau, x_{0}\right) .
\end{aligned}
$$

The last equality follows from the classical theorem for differentiating of the solution of differential equation on the initial condition (for example, see Theorem $1.16[9])$. Then from the equalities above and (3), we get

$$
\begin{aligned}
& \lim _{\Delta x_{i} \rightarrow} \frac{1}{\Delta x_{i}}\left(\Theta^{+}\left(x_{0}^{*}\right)-\Theta^{+}\left(x_{0}\right)\right) \\
= & -\lim _{\Delta x_{i} \rightarrow 0} \frac{\left\langle a, e_{i}\right\rangle}{\left\langle a, f\left(x\left(\theta ; x_{0}^{*}\right)\right)\right\rangle} \\
& -\lim _{\Delta x_{i} \rightarrow 0} \frac{\left\langle a, \int_{0}^{\Theta^{+}\left(x_{0}\right)} \frac{\partial}{\partial x} f\left(x\left(\tau ; x_{0}+\mu \cdot \Delta_{i} \cdot e_{i}\right)\right) \cdot \frac{1}{\Delta x_{i}}\left(x\left(\tau ; x_{0}^{*}\right)-x\left(\tau ; x_{0}\right)\right) d \tau\right\rangle}{\left\langle a, f\left(x\left(\theta ; x_{0}^{*}\right)\right)\right\rangle} \\
= & -\frac{\left\langle a, e_{i}\right\rangle+\left\langle a, \int_{0}^{\Theta^{+}\left(x_{0}\right)} \frac{\partial}{\partial x} f\left(x\left(\tau ; x_{0}\right)\right) \cdot \frac{\partial}{\partial x_{0 i}} x\left(\tau ; x_{0}\right) d \tau\right\rangle}{\left\langle a, f\left(x\left(\Theta^{+}\left(x_{0}\right) ; x_{0}\right)\right)\right\rangle} .
\end{aligned}
$$


Thus, we have shown that there exists

$$
\frac{\partial}{\partial x_{0 i}} \Theta^{+}\left(x_{0}\right), i=1,2, \ldots, n,
$$

and the conditions (2) are satisfied.

The theorem is proved.

\section{References}

[1] H. Akca, R. Alassar, V. Covachev, Z. Covacheva, E. Al-Zahrani, Continuous-time additive Hopfield-type neural networks with impulses, $J$. of Mathematical Analysis and Applications, 290, No. 2, (2004), 436-451.

[2] D. Bainov, A. Dishliev, The phenomenon beating of the solutions of impulsive functional differential equations, Communications in Applied Analysis, 1, No. 4, (1997), 435-442.

[3] R. Chukleva, A. Dishliev, K. Dishlieva, Stability of the differential equations with variable structure and no fixed impulsive moments using sequences of Lyapunov's functions, International J. of Differential Equations and Applications, 11, No. 1 (2012), 57-80.

[4] A. Dishliev, D. Bainov, Continuous dependence of the solution of a system of differential equations of a system of differential equations with impulses on the impulse hypersurfaces, J. of Mathematical Analysis and Applications, 135, No. 2, (1988), 369-382.

[5] A. Dishliev, K. Dishlieva, Continuous dependence of the solutions of differential equations under "short" perturbations on the right-hand side, Communications in Applied Analysis, 10, No. 2-3 (2006), 149-160.

[6] K. Dishlieva, A. Dishliev, A. Antonov, Periodic solutions of a model of Lotka-Volterra with variable structure and impulses, J. of Advances in Mathematics, 11, No. 6, (2015), 5317-5325.

[7] K. Dishlieva, A. Dishliev, A. Antonov, Totally reachable sets for autonomous systems of differential equations, International J. of Science, Technology and Management, 4, No. 6, (2015), 36-46.

[8] K. Dishliev, A. Dishliev, A. Antonov, R. Chukleva, Function of reachability for autonomous systems of differential equations, Mathematical Sciences Letters, 4, No. 2, (2015), 91-99. 
[9] A. Fillatov, The averaging methods in the differential and integraldifferential equations, Fan, Tashkent, 1971.

[10] H.-F. Huo, Existence of positive periodic solutions of a neutral delay LotkaVolterra system with impulses, Computers and Mathematics with Applications, 48, No. 12, (2004), 1833-1846.

[11] X. Li, H. Akca, X. Fu, Uniform stability of impulsive infinite delay differential equations with applications to systems with integral impulsive conditions, Applied Mathematics and Computation, 219, No. 14, (2013), 7329-7337.

[12] Y. Liu, W. Ge, Stability theorems and existence results for periodic solutions of nonlinear impulsive delay differential equations with variable coefficients, Nonlinear Analysis: Theory, Methods and Applications, 57, No. 3, (2004), 363-399.

[13] S. Nenov, D. Bainov, Impulsive dynamical systems, Proceedings of the Second International Colloquium on Differential Equations, (1992), 145166.

[14] S. Petkova, A. Antonov, R. Chukleva, Reachable sets for autonomous systems of differential equations and their topological properties, American J. of Applied Mathematics, 1, (2013), 49-54. 
